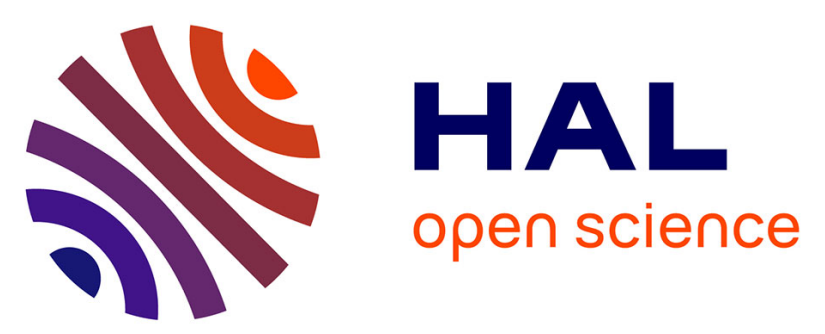

\title{
Hamiltonian stochastic processes induced by successive wave-particle interactions in stimulated Raman scattering
}

\author{
Alain Ghizzo, Daniele del Sarto, Thierry Reveille
}

\section{To cite this version:}

Alain Ghizzo, Daniele del Sarto, Thierry Reveille. Hamiltonian stochastic processes induced by successive wave-particle interactions in stimulated Raman scattering. Physical Review E: Statistical, Nonlinear, and Soft Matter Physics, 2009, 79 (4), pp.046404. 10.1103/PhysRevE.79.046404 . hal00524426

\section{HAL Id: hal-00524426 \\ https://hal.science/hal-00524426}

Submitted on 30 May 2018

HAL is a multi-disciplinary open access archive for the deposit and dissemination of scientific research documents, whether they are published or not. The documents may come from teaching and research institutions in France or abroad, or from public or private research centers.
L'archive ouverte pluridisciplinaire HAL, est destinée au dépôt et à la diffusion de documents scientifiques de niveau recherche, publiés ou non, émanant des établissements d'enseignement et de recherche français ou étrangers, des laboratoires publics ou privés. 


\title{
Hamiltonian stochastic processes induced by successive wave-particle interactions in stimulated Raman scattering
}

\author{
A. Ghizzo, D. Del Sarto, and T. Reveille \\ L.P.M.I.A., EPCS Nancy University, UMR 7040, BP 239, 54506 Vandoeuvre les Nancy, Cedex, France
}

(Received 5 September 2008; revised manuscript received 23 December 2008; published 14 April 2009)

\begin{abstract}
The long-time dynamics of particles interacting resonantly with large-amplitude coherent plasma wave is investigated in the kinetic regime of stimulated Raman scattering in which particle trapping plays a major role (and which corresponds to a high value of the parameter $k_{\mathrm{EPW}} \lambda_{D}$, where $k_{\mathrm{EPW}}$ is the plasma wave vector and $\lambda_{D}$ is the electron Debye length). Using Vlasov simulations, the dynamics of such particles become stochastic when repeated wave-particle interactions take place. For small values of the ratio $\tau_{\text {auto }} / \tau_{b}$ of the autocorrelation time to the bounce time of particle (condition usually met in backward propagation of the scattered wave) the turbulent regime results in the merging of phase-space trapping vortices according to a weak turbulencelike scenario. For high values of $\tau_{\text {auto }} / \tau_{b}$ (or narrow spectrum of longitudinal electric field as met when only one plasma wave is present), the stochasticity is now induced by particle trapping, detrapping, and retrapping in the adiabatically fluctuating field. The stochastic transitions performed by resonant particles above (or below) the separatrix limit in phase space determine now the long-time plasma evolution.
\end{abstract}

DOI: 10.1103/PhysRevE.79.046404

PACS number(s): 52.65.Ff, 52.35.Mw, 52.35.Ra, 52.38.Bv

\section{INTRODUCTION}

In stimulated Raman scattering, the scattered electromagnetic radiation may propagate either backward (denoted here SRS-B) or forward (SRS-F). In the weak Landau regime $\left[k_{\mathrm{EPW}} \lambda_{D} \lesssim 0.3\right.$ where $k_{\mathrm{EPW}}$ is the wave number of the Electron Plasma Wave (EPW) and $\lambda_{D}$ is the Debye length], it is well known that the main mechanism responsible for the saturation of SRS-B is the Langmuir decay instability (LDI) (see Ref. [1]), which is the interaction of primary and secondary EPWs with a resonant ion acoustic wave (IAW). The well-developed parametric theory based on the envelope model gives basic values of SRS instability threshold, growth rates in its initial stage, and provides some insight on the convective or absolute behavior of the SRS instability. However, in contemporary laser-plasma experiments, SRS has often displayed rich and complex physics, not predicted by the parametric theory such as the bursting behavior of reflectivity or anomalously low reflectivity, spectral gaps or broadening, as well as turbulent behaviors or the occurrence of secondary instabilities as the stimulated electron acoustic wave scattering involving low-frequency plasma modes.

In particular an inherent feature of strongly nonlinear SRS to transit from a coherent (regular) to chaotic dynamics was predicted by Skoric et al. in Ref. [2] using a classic threeoscillator model by increasing the quiver velocity. The simulation results of SRS-B exhibit a transition to lowdimensional wave chaos via spatiotemporal intermittency.

All in all, we would expect turbulence in a plasma subjected to strong wave-particle interactions to be very different from the conventional weak turbulence in particular when self-consistent interactions between waves and resonant particles are accurately taken into account. In particular in the regime where $k_{\mathrm{EPW}} \lambda_{D} \gtrsim 0.3$ (i.e., at high temperature or low density) referred usually as the kinetic regime of the SRS instability, for sufficiently strong drive, particle trapping plays a fundamental role, allowing the EPW to survive in spite of the high level of Landau damping (see, for instance,
Ref. [3]). This fact enhances the wave-particle interaction, making possible the occurring of an unusual somewhat chaotic state quite unlike classic weak turbulence and also quite unlike that obtained classic strong trapping or by the classic three-wave envelope model, i.e., which does not correspond to the classic wave chaos.

Semi-Lagrangian Vlasov-Maxwell simulations have, recently shown in Ref. [4], two new nonlinear behaviors: first there is a Morales-O'Neil plasma downshift (see Ref. [5]) retuned by a small wave-number shift which maintains the SRS-B resonance. The observed behavior corresponds to the combined action of the frequency shift of the amplified EPW induced by nonlinear trapping effect, compensated for a retuning of the wave number to maintain the parametric resonance over a long time. Second the simulations (in optical mixing, i.e., with probe injection at weak level) have shown that the main process that limits the continuation of the SRS-B instability by Langmuir wave frequency-wave-vector chirping and retuning is provided by a process which begins with pairwise vortex merging (VM) in phase space. This vortex-merging process then evolves toward a resonant broader spectrum resembling that of a weak electrostatic inverse cascade leading to a rather turbulent state. This is a possible scenario for the saturation of SRS-B. However, such a mechanism was never observed for SRS-F although SRS-F may also be characterized by trapping vortices in phase space. Although the empirical criterion of Bernstein-GreeneKruskal (BGK)-type merging [12] is now known fairly accurately, it is still unclear why exactly a pairwise trapping vortex merging is not observed in SRS-F.

On the other hand, the strong field limit is characterized by a narrow spectrum of the plasma field and the quasilinear approximation could produce incorrect results. This case corresponds to the limit $\tau_{b} \ll \tau_{\text {auto }}$, where $\tau_{b}$ $\sim \sqrt{m /\left(e E_{x, \text { max }} k_{\mathrm{EPW}}\right)}$ is the bounce time of the particles, deeply trapped in the electrostatic potential, and $E_{x, \max }$ being the maximum of the (longitudinal) electric field (here chosen in the $x$ longitudinal direction). The second time scale $\tau_{\text {auto }}$ 
$\sim 1 / k_{\mathrm{EPW}} \Delta v_{\varphi}$ is the field autocorrelation time, where $\Delta v_{\varphi}$ is the characteristic separation velocity range. In the weak turbulence limit we have then $\tau_{\text {auto }} \ll \tau_{b}$ or equivalently for a value $\tau_{\text {auto }} / \tau_{b} \ll 1$.

SRS-F is an example of such a process which may produce a coherent high-amplitude EPW capable to accelerate particles to relativistic velocities. In that case the longitudinal plasma field topography slowly changes and dynamics evolves quasiadiabatically. How could a diffusion process or a turbulent state be produced in simulation of SRS-F, which seems to lack of any broadening resonance character of the EPW, when only a quasimonochromatic wave exists (with no possible vortex merging)? There is, instead, an effective diffusion caused by the variation in wave amplitude and the resultant succession (for a few electrons) of trapping, detrapping, and retrapping, each event occurring near a phasespace separatrix. In particular the domain in phase space defined by the separatrix limits may fluctuate and particle trajectories may cross the instantaneous separatrices. Thus although SRS-F is also characterized by a strong trapping process, the vortex merging cannot be observed in that situation.

This paper presents some relevant numerical VlasovMaxwell simulations of the nonlinear evolution of the SRS instability in the new kinetic regime (i.e., $k_{\mathrm{EPW}} \lambda_{D} \geq 0.3$ ). The specificity of our approach consists in carrying out full kinetic Vlasov simulations of SRS-B and SRS-F and performing a statistical study of the stochastic transition through repeated wave-particle interaction cycles. To our knowledge there is no analysis including both electron trapping and turbulence effects of nonlinear and long-time evolution of stimulated scatter interactions in this "frontier" regime delimited by the $\tau_{\text {auto }} / \tau_{b}$ ratio. Both cases $\tau_{\text {auto }} / \tau_{b} \lesssim 1$ and $\tau_{\text {auto }} / \tau_{b} \gg 1$ were considered to study the formation of Hamiltonian stochasticity for SRS scenario. In this work, we present results obtained from simulations with a semiLagrangian relativistic Vlasov code. More details of the model may be found in Ref. [6].

\section{CAUSAL SIMULATION OF SRS-B IN THE WEAK TURBULENTLIKE REGIME}

For SRS-B, a new kinetic regime was recently identified by Kline et al. in Ref. [3]. At low $k_{B} \lambda_{D}$ value, LDI was observed via the occurring of multiple EPWs, while as $k_{B} \lambda_{D}$ increases, LDI was not observed and a single frequencybroadening EPW spectrum was observed. In that kinetic regime, LDI process is too heavily Landau damped to compete and particle trapping and the associated nonlinear frequency shift seem to play a fundamental role. A clear indication of such a kinetic behavior was the recent observation in Ref. [7] at the Trident laser facility of SRS-B of the possibility of low-frequency Electron Acoustic Wave (EAW) excitation. In underdense high-temperature plasmas, however, the Landau damping strongly limits SRS-B, which makes possible the growth of the forward scattering (for more details see Ref. [8]). In Ref. [9] we have investigated the coexistence of SRS-B and SRS-F. The parameter analysis predicts that there exists a fairly large region in the plasma parameter space where SRS-B and SRS-F may occur simultaneously.

When both of the Raman (SRS-F and SRS-B) processes occur simultaneously, there are three electromagnetic modes, i.e., the pump wave denoted by $\left(\omega_{0}, k_{0}\right)$, the backscattered mode $\left(\omega_{s B},-k_{s B}\right)$, and the forward scattered mode $\left(\omega_{s F}, k_{s F}\right)$. Two longitudinal electrostatic EPWs are then excited, $\left(\omega_{B}, k_{B}\right)$ and $\left(\omega_{F}, k_{F}\right)$, where the subscripts $B$ and $F$ refer to backward and forward scatterings, respectively. Electromagnetic waves verify the linear dispersion relations,

$$
\omega_{s F, s B, 0}^{2}=\omega_{p}^{2}+k_{s F, s B, 0}^{2} c^{2},
$$

while the electrostatic waves obeys the Bohm-Gross dispersion relation,

$$
\omega_{F, B}^{2}=\omega_{p}^{2}+3 k_{F, B}^{2} v_{\text {th }}^{2},
$$

where $\omega_{p}$ is the electron plasma frequency, $c$ is the light velocity in vacuum, and $v_{\mathrm{th}}=\sqrt{K_{B} T_{e} / m_{e}}$ is the thermal velocity of electrons. Equations (1) and (2), together with the following phase-matching conditions,

$$
\begin{aligned}
& \omega_{0}=\omega_{s B}+\omega_{B}=\omega_{s F}+\omega_{F}, \\
& k_{0}=-k_{s B}+k_{B}=k_{s F}+k_{F},
\end{aligned}
$$

determine the wave numbers and the frequencies of the different waves once the ratio of the electron density to the critical density $n_{0} / n_{c}$ and the electron temperature $T_{e}$ are given.

Let us begin with an example related to the kinetic regime of stimulated Raman backscattering dominated by weak plasma turbulence and nonlinear shift of EPW. We begin with a simulation of SRS-B (with the possible coexistence of SRS-B/SRS-F) in a homogeneous plasma with injected counterpropagating pump $\left(\omega_{0}, k_{0}\right)$ and seed $\left(\omega_{s B},-k_{s B}\right)$ light with intensities of $I_{\text {seed }}=10^{-4} I_{\text {pump }}$. The physical system consists of a plasma slab of $3900 \mathrm{c} / \omega_{0}$ length, surrounded by vacuum of $50 \mathrm{c} / \omega_{0}$ length on both sides. The total length of the system is $L=4096 \mathrm{c} / \omega_{0}$ corresponding to $625 \lambda_{0}$ (or $219 \mu \mathrm{m}$ with an incident pump wavelength of $\lambda_{0}$ $=0.351 \mu \mathrm{m}$ in vacuum). The quiver momentum of the pump wave is $a_{0}=0.025$ which corresponds to an intensity of $I_{\text {pump }} \simeq 7 \times 10^{15} \mathrm{~W} \mathrm{~cm}^{-2}$. The plasma density is normalized to the value of $n_{0} \simeq 0.0825 n_{c}$ for an electron temperature of $T_{e}=2 \mathrm{keV}$. Ions are kept fixed here to simplify the presentation but mobile ions do not change the physical process considered here (in particular we have carried out different simulations with mobile ions with a ratio of the ion mass to the electron mass of $m_{i} / m_{e}=1836$ and $T_{e}=10 T_{i}$ but LDI was not observed in simulation in that regime of instability; we have just noted the beginning of stimulated Brillouin backscattering which remains at weak level during the simulation).

Normalizing frequencies to the pump frequency $\omega_{0}$, we have used here for matching conditions of SRS-B, i.e., given by first part of Eqs. (3) and (4), the following quantities: $\omega_{s B}=2 / 3 \omega_{0}$ and $k_{s B} c / \omega_{0}=0.6016$ for the seed and $\omega_{B}$ $=1 / 3 \omega_{0}$ and $k_{B} c / \omega_{0}=1.5594$ for the EPW, which corresponds to a value of $k_{B} \lambda_{D} \simeq 0.34$ well into the kinetic regime of SRS-B. While the parameters of this case are indeed those of a peak density in [4], here we can isolate the local physics 

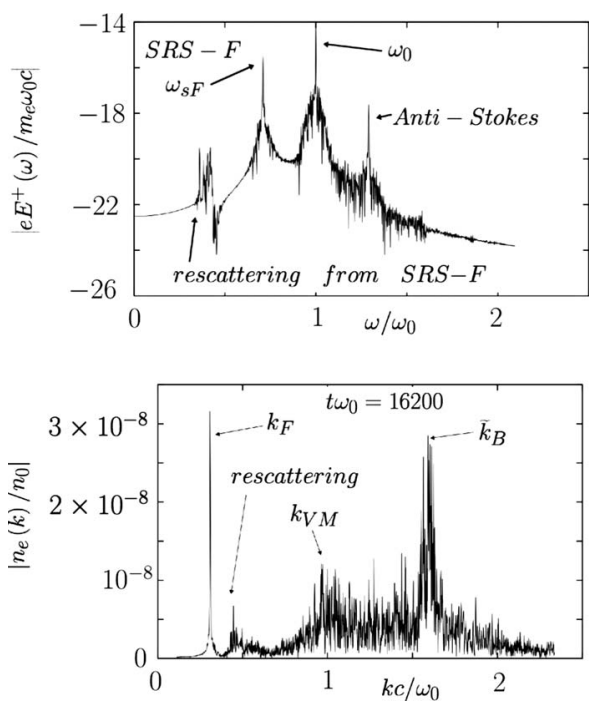

FIG. 1. Following numerical results of Fig. 3 for the uniform slab plasma, the frequency spectrum of the transmitted light (on a logarithmic scale) is shown in the top panel and the spectrum in wave vector of the longitudinal (plasma) field on a long time at $t \omega_{0}=16200$ (bottom panel).

in detail in a smaller uniform slab simulation, having verified in [4] the occurrence of the phenomena of interest in a realistic geometry.

Thus, as observed by others as given in Refs. [7,10,11] and in Vlasov simulations for parabolic profiles in Ref. [4] in the first phase of the instability, the plasma wave undergoes a frequency decrease, according to the basic idea of Morales and O'Neil (see Ref. [5]) regarding the effect of trapped electrons on the frequency of the Langmuir wave, while the wave vector increases slightly so as to maintain the SRS resonance (see, for instance, Ref. [4]). In Ref. [4] we have shown that the main process that limits the continuation of the SRS-B instability by EPW frequency or wave-number shift and retuning is provided by phase-space hole coalescence.

To summarize what happens in various regions of the plasma, let us consider the data of Fig. 1, showing the $\omega$-spectrum amplitude of the forward field $e E^{+} / m_{e} \omega_{0} c$ (top panel), the bottom panel corresponds to the $k$ spectrum of the longitudinal field $e E_{x} / m_{e} \omega_{0} c$ and shows what happens when SRS-F is now well established in the plasma at $t \omega_{0}$ $=16200$.

First as expected by Morales and O'Neil [5], the nonlinear shift in frequency induced by trapping effects was observed to have its upper limit value at $\omega_{s B}=0.75 \omega_{0}$ (rather than the original seed of $\left.\omega_{s B}=0.666 \omega_{0}\right)$ for the scattered light and the corresponding downshifted limit value from the density spectrum to be $\widetilde{\omega_{B}}=0.25 \omega_{0}$ for EPW rather than the original value of $\omega_{B}=0.333 \omega_{0}$ and well below the plasma frequency $\omega_{p}=0.2873 \omega_{0}$. We have denoted here $(\widetilde{\omega}, \widetilde{k})$ the new values of the frequency and wave number of the shifted wave. In particular the matching conditions of SRS-B resonance given by Eqs. (3) and (4), related to SRS-B, are now replaced by

$$
\omega_{0}=\widetilde{\omega_{s B}}+\widetilde{\omega_{B}}=\omega_{s B}\left(k_{0}-k_{B}-\delta k\right)+\omega_{B}-\delta \omega,
$$

$$
k_{0}=-\widetilde{k_{s B}}+\widetilde{k_{B}}=-\widetilde{k_{s B}}+k_{B}+\delta k .
$$

While in the scenario of Skoric et al. in Ref. [2] the transition of turbulence is made via spatial temporal intermittency, here trapping effects are important. This leads to the formation and growth of BGK-type phase-space holes (see, for instance, Ref. [12]), the result of vortex-merging process (at wave number close to $k_{\mathrm{VM}} c / \omega_{0} \sim 1$ ). These BGK-type structures are stable and self-sustained and may persist over a long time (several thousands of $\omega_{0}^{-1}$ ) after the pump was turned off. Rather than continuing the retuning three-wave resonance process indefinitely these resonant (and nonlinear) waves (characterized by trapping structures in phase space) begin to merge.

This second phase of the instability is now characterized by the occurring of secondary parametric instabilities as, for instance, rescattering of SRS-F light by the feature associated with the velocity merging (such as Ref. [4]) followed by the apparent inverse cascade process. Here the light was created by SRS-F, where the wave characteristics $\left(\omega_{s F}, k_{s F}\right)$ scatter off the kinetic or VM mode with parameters given by $\omega_{\mathrm{VM}}=0.25 \omega_{0}$ and $k_{\mathrm{VM}} c / \omega_{0} \sim 1$ into a backward-propagating light wave with frequency $\omega_{s}=0.46 \omega_{0}$ and $k_{s} c / \omega_{0} \simeq 0.36$. Frequency and wave vector matching are then $\omega_{s F}=\omega_{s}$ $+\omega_{\mathrm{VM}}$ and $k_{s F}=-k_{s}+k_{\mathrm{VM}}$. Here we have $\omega_{s F}=0.71 \omega_{0}$ and $k_{s F} c / \omega_{0} \simeq 0.65$ for the scattered SRS-F wave. The values of the corresponding EPW associated with SRS-F from the linear dispersion relation are then $\omega_{F}=0.289 \omega_{0}$ for the frequency and $k_{F} c / \omega_{0}=0.308$ for the wave vector.

Interestingly there is some evidence to suggest that the manner in which the chaotic behavior arises on a long time is linked to the occurring of secondary instabilities (rescattering of SRS-F light by BGK-type waves, rescattering of the probe light by EPW, generation of anti-Stokes scattering, coupling between SRS-F and SRS-B, etc.). That is to say, different EPWs or nonlinear waves are now generated which may indeed overlap in phase space and give rise to a turbulent state. We expect that such nonlinear behaviors arise in a plasma characterized by a small value of $\tau_{\text {auto }} / \tau_{b}$ but not too far of 1 $\left(\tau_{\text {auto }} / \tau_{b} \lesssim 1\right)$.

Here the bounce time of trapped particles, which may be given by $\tau_{b}=\sqrt{m_{e} / e E_{x, \max } k_{B}}$, is long compared to the other relevant time scales. In particular the field autocorrelation time $\tau_{\text {auto }} \sim 1 / k_{B} \Delta v_{\varphi} \ll \tau_{b}$, where $\Delta v_{\varphi}$ is the characteristic separation between maximum and minimum phase velocities. The plasma is found in a weak turbulence regime with a $\tau_{\text {auto }} / \tau_{b}$ parameter given by

$$
\tau_{\text {auto }} / \tau_{b}=\frac{1}{\Delta \omega} \sqrt{\frac{e E_{x, \max } k_{B}}{m_{e}}},
$$

where we have used the relation $\Delta v_{\varphi}=\Delta \omega / k_{B}$. Thus an estimation of the $\tau_{\text {auto }} / \tau_{b}$ ratio gives $\left(\omega_{0} / \Delta \omega\right) \sqrt{\left(e E_{x, \max } / m_{e} \omega_{0} c\right)\left(k_{B} c / \omega_{0}\right)}$, i.e., $\sqrt{0.01 \times 1.56 / 0.3}$ $\simeq 0.4$ for this simulation.

\section{PERIODIC SIMULATIONS FOR TURBULENT PLASMA STATE CHARACTERIZED BY $\tau_{\text {auto }} / \tau_{b} \lesssim 1$ : PHASE SPACE VORTEX MERGING AND SECONDARY INSTABILITIES}

While the causal (or open) Vlasov simulations are necessary to understand realistic cases, these simulations, as seen 
in Sec. II, are also difficult to analyze in detail. One of the difficulties is that even the simplest three-wave instability involves coupled nonlinear partial differential equations in both time and space. These simulations introduce two different types of nonlinearities induced by wave coupling or by kinetic effects (trapping, etc.). After a time, nonlinear wave coupling, wave chaos or cascade, and secondary parametric instabilities arise. In order to build a physical picture of what happens in plasma turbulence, it is perhaps more fruitful to return to the periodic case, which allows one to separate the different instabilities.

In the three-wave parametric decay from the pump to the backscattered (here denoted by $s B$ ) to the EPW, we have then in a periodic situation

$$
\omega_{0}\left(k_{0}\right)+\delta \omega=\omega_{s B}\left(k_{s B}\right)+\omega_{B}\left(k_{B}\right), \quad k_{0}=-k_{s B}+k_{B},
$$

where we assumed perfect $k$ matching (since we have a periodic simulation box and match mode numbers exactly) while we introduce $\delta \omega$ for frequencies which contains the mismatch (if any).

Because we eventually wish to discuss action conservation (see Ref. [13] for more details), we found it convenient to recall the fluid model in terms of action amplitude $a_{0, s B, B}$ for SRS-B (defined such that the action density $S=a a^{*}$ is given from the energy density $W$ by $S=W / \omega)$,

$$
\begin{aligned}
& \left(\frac{\partial}{\partial t}+v_{g, 0} \frac{\partial}{\partial x}\right) a_{0}=-\Lambda a_{s B} a_{B} e^{i \delta \omega t}, \\
& \left(\frac{\partial}{\partial t}+v_{g, s B} \frac{\partial}{\partial x}\right) a_{s B}=\Lambda a_{0} a_{B}^{*} e^{-i \delta \omega t}, \\
& \left(\frac{\partial}{\partial t}+v_{g, B} \frac{\partial}{\partial x}\right) a_{B}=\Lambda a_{0} a_{s B}^{*} e^{-i \delta \omega t},
\end{aligned}
$$

where $a_{i}=A_{i} \sqrt{\epsilon_{0} \omega_{i} / 2}$ for $i=0, s B$ and $a_{B}=\omega_{p}^{-1} E_{B} \sqrt{\epsilon_{0} \omega_{B} / 2}$; $A_{0}$ and $A_{s B}$ being the corresponding amplitude of the pump and scattered mode of the potential vector and $E_{B}$ being the amplitude of the envelope of the longitudinal electric field. Here the coupling coefficient is $\Lambda$ $=\left(e / 2 m_{e}\right)\left(2 \epsilon_{0} \omega_{0} \omega_{s B} \omega_{B}\right)^{-1 / 2} k_{B} \omega_{p}$. In a periodic system, we can drop the $\partial / \partial x$ term in Eqs. (9)-(11), and after a little algebra, we can obtain the usual Manley-Rowe relations,

$$
\begin{gathered}
C_{s B}(t)=S_{0}+S_{s B}=\left|a_{0}\right|^{2}+\left|a_{s B}\right|^{2}=\mathrm{const}=C_{s B}(t=0), \\
C_{B}(t)=S_{0}+S_{B}=\left|a_{0}\right|^{2}+\left|a_{B}\right|^{2}=\mathrm{const}=C_{B}(t=0) .
\end{gathered}
$$

The system chooses among two kinds of diffusive chaotic behaviors according to the value of the ratio $\tau_{\text {auto }} / \tau_{b}$. We focus here on the case $\tau_{\text {auto }} / \tau_{b} \lesssim 1$. It is well known that weak turbulence corresponds usually to the $\tau_{\text {auto }} / \tau_{b} \ll 1$ regime. A more rigorous picture for the $\tau_{\text {auto }} / \tau_{b} \lesssim 1$ regime can be given, which includes weak turbulence, as the result of the vortex merging as an essential ingredient of plasma turbulence in the kinetic regime.

We begin with a simulation with similar physical parameters as those used in Sec. II. Our $x-p_{x}$ phase-space grid sampling was set as $256 \times 1024$ and our time step was taken to be $0.02 \omega_{p}^{-1}$, while $p_{x, \max } / m_{e} c$ was chosen to be 1 . The velocities are normalized to the light velocity $c$ and frequencies to the plasma frequency $\omega_{p}$ (which is the conventional choice for periodic or uniform plasmas). The electromagnetic pump wave mode number has been chosen to $3 \Delta k$ (and $\Delta k$ $=2 \pi / L$ being the fundamental mode), the choice of $k_{0}$ in effect determines the plasma length $L$ in terms of $c / \omega_{p}$. For a plasma temperature of $T_{e}=2 \mathrm{keV}$, a good frequency match was obtained by choosing $k_{0} c / \omega_{p}=3.6$ (and so $k_{s B} c / \omega_{p}$ $=2.4$, i.e., $k_{s B}=2 \Delta k$ and $k_{B} c / \omega_{p}=6$ or $\left.k_{B}=5 \Delta k\right)$. The fundamental wave vector is then $\Delta k=1.20 \omega_{p} c^{-1}$. The corresponding frequencies are then, using the linear dispersion relation, $\omega_{0} \simeq 3.736 \omega_{p}$ for the pump wave (i.e., a ratio of the electron density to the critical density of $n_{0} / n_{c}=\omega_{p}^{2} / \omega_{0}^{2} \simeq 0.071$ ), $\omega_{s B} \simeq 2.60 \omega_{p}$ for the backscattered wave, and $\omega_{B} \simeq 1.192 \omega_{p}$ for the EPW induced by SRS-B.

This simulation corresponds to the weak turbulent regime of the instability with a parameter $\tau_{\text {auto }} / \tau_{b}$ of $\left(\omega_{p} / \Delta \omega\right) \sqrt{\left(e E_{x, \max } / m_{e} \omega_{p} c\right)\left(k_{B} c / \omega_{p}\right)} \quad$ or $\sqrt{0.04 \times 6 / 0.5}$ $\simeq 0.975$, i.e., $\tau_{\text {auto }} / \tau_{b} \sim 1$. Here we have for the frequency width $\Delta \omega / \omega_{p} \sim 1$.

Here we have a frequency mismatch given by $\delta \omega$ $\simeq 0.05 \omega_{p}$. For our simulations, we take out $\left(\omega_{p}, c\right)$ normalized pump electric field $e E_{0} / m_{e} \omega_{p} c$ to be 0.149 , i.e., a somewhat higher value in comparison with the value used in Sec. II. The action is normalized by division with $n_{0} m_{e} c^{2} / \omega_{p}$, so the normalized pump action density is $S_{0} \omega_{p} / n_{0} m_{e} c^{2}$ $=\left(2 \omega_{p} / \omega_{0}\right)\left(e E_{0} / m_{e} \omega_{p} c\right)^{2}$, i.e., 0.00298 .

Numerical results are shown in Fig. 2. The left top panel shows the electromagnetic spectrum in frequency of $e E_{y} / m_{e} \omega_{p} c$. The figure shows that the expected values are obtained with high accuracy: the pump frequency is observed at frequency $\omega_{0} \simeq 3.75 \omega_{p}$, in good agreement with the theoretical value of $\omega_{0}=1 / \sqrt{n_{0} / n_{c}}=3.736 \omega_{p}$. The backscattered mode frequency is close to $\omega_{s B} \simeq 2.58 \omega_{p}$ (while the exact value obtained via the linear dispersion relation is $\left.\omega_{s B}=2.60 \omega_{p}\right)$. The signature of backward Raman scattering of the probe beam is also clearly visible at the frequency $\omega_{2 s B} \simeq 1.54 \omega_{p}$. This is due to a secondary instability taking place: the probe light even if somewhat mismatched can couple with the plasma to generate a backscattered mode, i.e., a counterpropagating electromagnetic wave $\left(\omega_{2 s B}, k_{2 s B}\right)$, which propagates in the opposite direction of the probe beam and which is resonant in frequency and off resonance in wave number, according to the relations

$$
\omega_{s B}=\omega_{2 s B}+\omega_{2 B}, \quad k_{s B}=-k_{2 s B}+k_{2 B} .
$$

From the linear dispersion relation, we have $\omega_{2 s B}=\left(\omega_{p}^{2}\right.$ $\left.+k_{2 s B}^{2} c^{2}\right)^{1 / 2} \simeq 1.562 \omega_{p}$, using a value of $k_{2 s B} c / \omega_{p}=1.2$, for the scattered wave vector and $\omega_{2 B}=\left(\omega_{p}^{2}+3 k_{2 B}^{2} v_{\mathrm{th}}^{2}\right)^{1 / 2} \simeq 1.0734 \omega_{p}$ and $k_{2 B} c / \omega_{p}=3.60$ [i.e., mode 3 or $(3 \Delta k) c / \omega_{p}$ ] for the second EPW, leading to a mismatch in frequency close to $\delta \omega$ $=\omega_{s B}-\omega_{2 s B}-\omega_{2 B} \simeq-0.035 \omega_{p}$. The electrostatic spectrum in frequency is plotted in the top panel in Fig. 2 as a function of $\omega / \omega_{p}$ showing that the spectrum begins to broaden in spite of the fact the system is here assumed to be periodic. Here EPWs undergo a slight frequency decrease due to nonlinear 

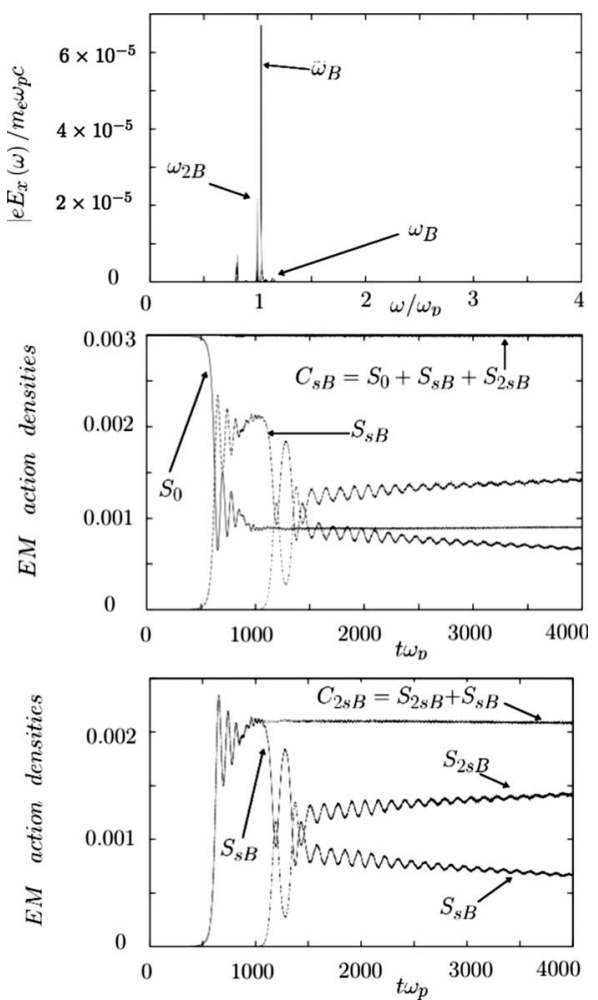

FIG. 2. Periodic Vlasov simulation for SRS-B scenario with cascade process or rescattering of the amplified probe giving rise to two different Langmuir waves. Top panel: electrostatic spectrum in frequency showing that EPW frequencies were downshifted due to strong trapping effects. Middle panel: temporal behavior of the three electromagnetic waves $S_{0}$ (pump), $S_{s B}$ (amplified probe), and $S_{2 s B}$ and their mutual sum. Bottom panel: main actors in the rescattering of the probe.

particle trapping effects (as met in the case of an open system in Sec. III).

The middle and bottom panels in Fig. 2 illustrate and confirm the excitation of both parametric three-wave instabilities. We have plotted the temporal behavior of the electromagnetic action densities $S_{0}$ (for the pump), $S_{s B}$ (for the scattered wave induced by SRS-B of the pump), and $S_{2 s B}$ (for SRS-B of the probe light) and their mutual sum $C_{s B}=S_{0}$ $+S_{s B}+S_{2 s B}$. One can see clearly that taking into account the $S_{2 s B}$ term indeed gives a total $C_{s B}$ which is well conserved during the simulation. The general behavior is as expected from the canonical parametric oscillator decay model: nonlinear and decaying oscillatory transfer from pump to daughters and back again. Note that this action sum for the basic backscattering SRS has already been reported in Ref. [14]. However the situation is somewhat more complex due to the "cascade" process of the probe beam which appears here as a secondary instability at $t \omega_{p}=1200$. The bottom panel shows the time behavior of the sum $C_{2 s B}=S_{s B}+S_{2 s B}$ (we have also plotted again the modes $S_{s B}$ and $S_{2 s B}$ ) indicating clearly that now the probe behaves as a new pump wave allowing action transfer from the amplified probe to daughter waves [scattered mode $\left(\omega_{2 s B}, k_{2 s B}\right)$ and the second Langmuir wave $\left.\left(\omega_{2 B}, k_{2 B}\right)\right]$.

To aid in the understanding of what is happening, we present in Fig. 3 snapshots of the electron distribution func-

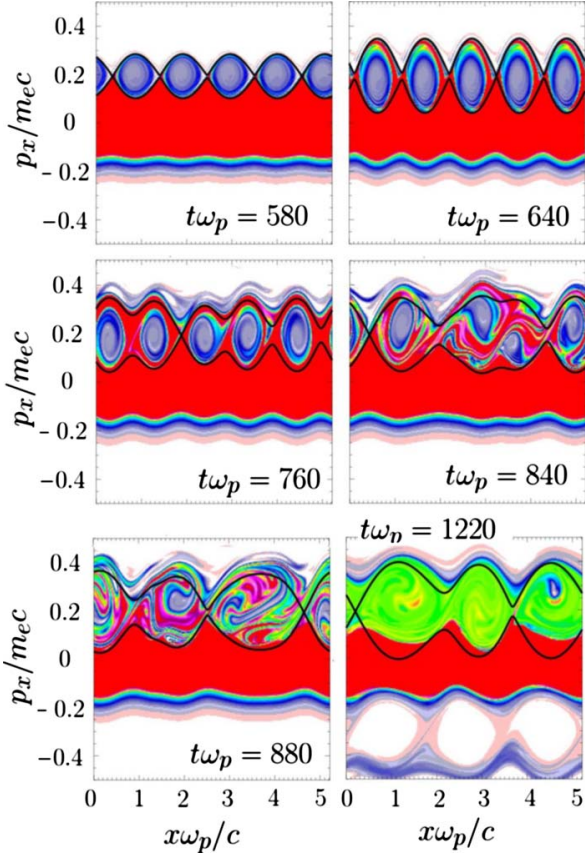

FIG. 3. (Color online) For a periodic simulation, phase-space snapshots showing trapping structures moving at the phase velocity $v_{\varphi B}=\omega_{B} / k_{B}$. We have also superimposed the separatrix limits directly obtained through the data of the electrostatic potential computed by the Vlasov code. Top panels: formation of the very neat phase-space hole wave train (here five holes corresponding to the mode $k_{B}=5 \Delta k$ ) induced by SRS-B. Next three snapshots show the coalescence which begins with the symmetry breaking leading to a more turbulent state. Last curve shows the rescattering of the amplified probe.

tion in phase-space $\left(p_{x} / m_{e} c\right.$ versus $\left.x \omega_{p} / c\right)$ plot for various times during the plasma evolution. We have also introduced the separatrix limits. These separatrices can be very simple if we assumed that only one EPW is generated into the plasma by SRS-B and that this EPW is stationary in a frame moving at the phase velocity of the plasma wave $v_{\varphi B}=\omega_{B} / k_{B}$. The motion of a test particle is then obtained from the Hamiltonian in the laboratory frame (for a given potential),

$$
H_{\mathrm{lab}}=e \Phi_{\mathrm{lab}}\left(x-v_{\varphi B} t\right)+m_{e} c^{2}(\gamma-1),
$$

where $\Phi_{\text {lab }}$ data are directly obtained by our Vlasov code. The new Hamiltonian in the frame of the plasma wave may be calculated from the Lorentz transform in the following form:

$$
H_{w}=\gamma_{\varphi B}\left[e \Phi_{\mathrm{lab}}\left(x-v_{\varphi B} t\right)+m_{e} c^{2}(\gamma-1)-v_{\varphi B} p_{x}\right] .
$$

Here $\gamma_{\varphi B}$ is the Lorentz factor corresponding to the phase velocity. From code data, we may obtain the maximum potential amplitude $\Phi_{0, \text { lab }}$ and a little algebra yields to the expression of the Hamiltonian at the $X$ points (where we have indeed $\left.\gamma=\gamma_{\varphi B}\right)$, which takes the form

$$
H_{w}^{s}=\gamma_{\varphi B}\left[e \Phi_{0, \mathrm{lab}}+m_{e} c^{2}\left(\gamma_{\varphi B}^{-1}-1\right)\right] .
$$


If $H_{w}<H_{w}^{s}$ the particle is trapped, while for the opposite inequality, it is of course not trapped. Thus from the separatrix orbit condition, one can readily solve the resulting quadratic equation and obtain

$$
\frac{p_{x \pm}(x)}{m_{e} c}=u_{\varphi B}\left(1+\frac{e \gamma_{\varphi B}}{m_{e} c^{2}} \Delta \Phi\right) \pm \sqrt{\left(1+\frac{e \gamma_{\varphi B}}{m_{e} c^{2}} \Delta \Phi\right)^{2}-1}
$$

where $u_{\varphi B}=\gamma_{\varphi B} \beta_{\varphi B}$, with $\beta_{\varphi B}=v_{\varphi B} / c$, and $\Delta \Phi=\Phi_{0 \text {, lab }}$ $-\Phi(x)$.

In Fig. 3, we present the separatrices calculated in this way from the simulation electrostatic potential superimposed on the very accurate phase-space representation afforded by our Vlasov code. The top panel in Fig. 3 (at times $t \omega_{p}$ $=580$ and $\left.t \omega_{p}=640\right)$ clearly exhibits the trapping and formation of holes, with a normalized phase velocity of $\beta_{B \varphi}$ $=0.198$ or equivalently a phase momentum $p_{B \varphi} / m_{e} c$ $=\beta_{B \varphi} / \sqrt{1-\beta_{B \varphi}^{2}} \simeq 0.203$ for the EPW $\left(\omega_{B}, k_{B}\right)$ in the initial phase of the instability, with a dominant mode $k_{B}=5 \Delta k$.

The middle panels in Fig. 3 (i.e., at times $t \omega_{p}=760$ and $\left.t \omega_{p}=840\right)$ exhibit the coalescence of trapping vortices with the symmetry breaking of an apparently random pair of vortices. This vortex merging is thus directly linked to the occurrence of the cascade process. At $t \omega_{p}=1200$ the coalescence is finished and the rescattering of the probe takes place. This vortex merging, met in an inhomogeneous version of the problem, seems to be similar with that observed in electrostatic simulations observed in the very two-stream first vortex merger work of Bertrand and co-workers in Refs. [15] or [16] leading to structures similar to BGK waves. This pairwise vortex merging was also observed in Ref. [4] in the case of a more realistic parabolic profile and proves to be pertinent even though we are faced with a larger open system and the persistence of the holes was observed over some thousands of inverse plasma frequency. In Ref. [4] we have shown that it is this process which is responsible of the SRS-B saturation and stop the EPW frequency shift. At time $t \omega_{p}=880$ (bottom left panel in Fig. 3) a rather turbulent state is now observed and this lack of coherence is what first breaks the resonant SRS coupling. The plasma is however also subject to the decay of the probe electromagnetic wave, as can be seen in the last bottom panel in Fig. 3 at time $t \omega_{p}=1220$. Since the probe beam is propagating from the right to the left in Fig. 3, the second EPW exhibits a negative phase-space velocity $v_{\varphi 2 B}=-\omega_{2 B} / k_{2 B}$ and we now observe the phase-space holes at phase momentum $p_{\varphi 2 B} / m_{e} c$ $\simeq-0.31$. Thus rescattering of SRS-B light (probe light) by a Raman backscattering limits also the growth of the EPW associated with SRS-B. As a consequence, the forwardpropagating Langmuir wave will remain at weaker level and will not trap as many electrons nor accelerate them to such a high energy. We must also point out that the occurrence of the second EPW (also located on the mode $3 \Delta k c / \omega_{p}$ but with negative phase velocity) leads to the breakdown of the orbit model since more than one EPW is excited.

\section{CONNECTION WITH STANDARD EPW OVERLAPPING: SRS-S AND SRS-F COUPLING}

The particle trajectories may become chaotic in momentum space when the trapping regions of the different Fourier
TABLE I. Wave numbers and frequencies of plasma wave and scattered Stokes modes for SRS-B and SRS-F.

\begin{tabular}{llcc}
\hline \hline & $\omega / \omega_{p}$ & $k c / \omega_{p}$ & Fourier mode \\
\hline Pump wave $E_{0}$ & 2.380 & 2.16 & 8 \\
Stokes wave in SRS-B $E_{s B}$ & 1.1368 & 0.54 & 2 \\
EPW in SRS-B $E_{B}$ & 1.1949 & 2.70 & 10 \\
Stokes wave in SRS-F $E_{S F}$ & 1.2868 & 0.81 & 3 \\
EPW in SRS-F $E_{F}$ & 1.0521 & 1.35 & 5 \\
\hline \hline
\end{tabular}

modes of the longitudinal electric field overlap significantly. This case differs from the pairwise vortex-merging scenario, met in Sec. III, where only one EPW is present. One must distinguish between the ordinary and uninteresting forced coalescence when one wave succeeds another of differing wave number and the spontaneous and symmetry-breaking sporadic coalescence such as that of Fig. 3 in Sec. III.

We give here an example where SRS-B and SRS-F may occur simultaneously, relevant to the kinetic regime of SRS instability. Initially the plasma is Maxwellian with a thermal velocity of $v_{\text {th }} / c=0.139$, corresponding to an electron temperature of $T_{e}=10 \mathrm{keV}$, well inside the kinetic regime of SRS-B. This simplified model makes it possible to study, with spatial and temporal resolutions, the interplay between wave-particle dynamics and the nonlinear fluid behavior of both EPWs, without the complications of physics introduced by the plasma-vacuum interface. The frequency of the pump wave is $\omega_{0}=2.38 \omega_{p}$ (i.e., a ratio of $n_{0} / n_{c}=0.176$ ) and the amplitude of the quiver momentum is here $a_{0}=0.10$. The plasma parameters given above are in the optimum operating region for simultaneous SRS-B and SRS-F as predicted in Ref. [17].

These parameters allow us to study the possibility of EPW overlap. The frequencies and the wave vectors of the scattered electromagnetic and electrostatic waves can be solved from dispersion relations (1) and (2) and phasematching conditions (3) and (4). The wave numbers and the frequencies of the various waves are given in Table I.

The corresponding matching errors introduced by the spatial periodicity are then $\delta \omega_{B}=\omega_{0}-\omega_{s B}-\omega_{B} \simeq 0.048 \omega_{p}$ for SRS-B and $\delta \omega_{F}=\omega_{0}-\omega_{s F}-\omega_{F} \simeq 0.041 \omega_{p}$ for SRS-F. The phase velocities (momenta) of the plasma waves are $v_{\varphi B}$ $=\omega_{B} / k_{B} \simeq 0.442 c$ (the corresponding phase momentum being $\left.p_{\varphi B} \simeq 0.493 m_{e} c\right)$ and $v_{\varphi F}=\omega_{F} / k_{F} \simeq 0.779 c \quad$ and $p_{\varphi F}$ $\simeq 1.24 m_{e} c$ for the phase momentum). For SRS-B the value of $k_{B} \lambda_{D}$ is equal to 0.375 , well into the kinetic regime, while $k_{F} \lambda_{D} \simeq 0.187$ is localized in a fluid-kinetic transition regime for the EPW connected to SRS-F. The electromagnetic (top panel) and electrostatic (shown in the middle panel) spectra are illustrated in Fig. 4. The narrow peaks in both spectra are visible and are located in good agreement with the theoretical values calculated from the phase-matching conditions (see Table I). However when nonlinear effects become important, the electrostatic spectrum broadens considerably reaching a down-shifted limit value of $\widetilde{\omega_{B}} \simeq 0.83 \omega_{p}$ (by the Morales-O'Neil shift [5]), i.e., well below the plasma frequency. The electromagnetic action densities are plotted on 

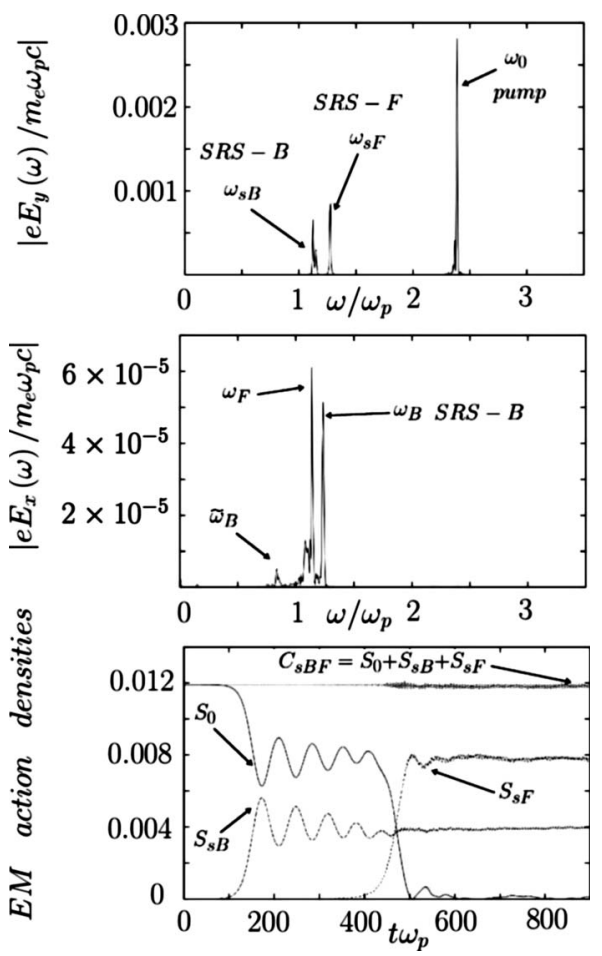

FIG. 4. For a periodic Vlasov simulation in simultaneous SRS-B and SRS-F coupling in the kinetic regime, electromagnetic spectrum in frequency in the top panel showing the three modes $\omega_{0}$ (pump), $\omega_{s B}$ (Stokes mode for SRS-B), and $\omega_{s F}$ (Stokes mode for SRS-F). The corresponding electrostatic spectrum is shown in the middle panel and the corresponding action densities in the bottom panel. Numerical parameters are those presented in Table I.

the bottom panel in Fig. 4. The temporal separation of both SRS instabilities is very clear. SRS-B is produced at the earlier time before the onset of forward scattering light amplification. At $t \omega_{p}=400$ it is necessary to take into account the contribution of the mode $S_{S F}=a_{S F} a_{S F}^{*}$ into the calculation of the total electromagnetic action density $C_{s B F}=\left|a_{0}\right|^{2}+\left|a_{s B}\right|^{2}$ $+\left|a_{S F}\right|^{2}$ to obtain a very good conservation of this ManleyRowe invariant. Note also the total depletion of the pump wave after time $t \omega_{p}=400$. In Fig. 5, the particle trapping induced by SRS-B is illustrated by a phase-space plot at time $t \omega_{p} \simeq 252$. At time $t \omega_{p}=360$ we observe a more complex plasma behavior induced by the presence of both EPWs, produced by SRS-B and SRS-F processes. What has happened, in the beginning, is that the coherent plasma wave structures of the forward-going plasma wave (here mode 10), produced by SRS-B, after about $t \omega_{p}=150$, are now superseded by the forward-going plasma wave (mode five) produced by SRS-F on the remaining pump (about $2 / 3$ of the original pump value) after about $t \omega_{p}=150$. Note that the conversion to forward-going scattered electromagnetic mode is more or less complete by about $t \omega_{p}=500$. However the destabilization of the primary EPW, produced by SRS-B, is now realized and induced by the seed and growth of SRS-F instability. This destabilization leads to a symmetry-breaking and pairwise vortex merging at time $t \omega_{p} \simeq 522$.

The corresponding $\tau_{\text {auto }} / \tau_{b}$ ratio is here $\left(\omega_{p} / \Delta \omega\right) \sqrt{\left(e E_{x, \max } / m_{e} \omega_{p} c\right)\left(k_{B} c / \omega_{p}\right)}$ close to $\sqrt{0.05 \times 2.68} /$

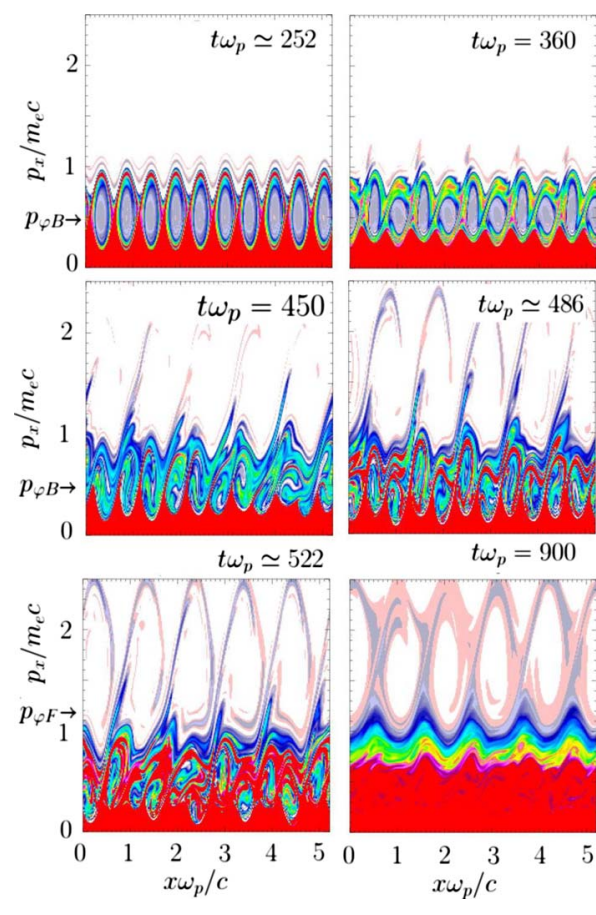

FIG. 5. (Color online) Phase space representation of the electron distribution function in the case of the numerical simulation shown in Fig. 4 (SRS-B and SRS-F coupling). Here Raman backscattering enhances the wave-particle interaction and intensifies particle trapping.

$0.5 \simeq 0.73$. The wave-particle interaction in simultaneous SRS-B and SRS-F is strong in spite of the large phase momentum of the SRS-F plasma wave (here $p_{\varphi F} \simeq 1.24 m_{e} c$ ). The reason for this is Raman backscattering, which enhances the wave-particle interaction and intensifies particle trapping. At time $t \omega_{p} \simeq 486$, SRS-F begins to occur and we may observe the corresponding phase-space vortices induced by trapping effects in phase space. Finally at time $t \omega_{p} \simeq 900$, SRS-F dominates now into plasma and rather coherent structures are observed. What is really happening is that SRS-F may be continually intensified through the distortion of the electron distribution function in velocity around the phase velocity $v_{\varphi B}$ due to the first trapping mechanism induced by SRS-B. This takes the form of a second particle trapping mechanism around the higher phase velocity $v_{\varphi F}$ since there are now enough fast resonant particles which may interact with the second EPW. The end result is then a net transfer of action from the pump to fast particles.

\section{WAVE-PARTICLE INTERACTION FOR HIGH $\tau_{\text {auto }} / \tau_{b}$ VALUES: PARTICLE TRAPPING-DETRAPPING OR RETRAPPING SCENARIO}

The strong turbulence limit $\tau_{b} \ll \tau_{\text {auto }}$ is usually characterized by narrow electrostatic spectrum both in wave number and frequency, so many bounce cycles may be realized by a test particle before phase modifications are produced. A chaotic behavior was also observed in numerical simulations where no EPW overlapping was possible since just a single EPW was present in system. Many questions remain unan- 
swered even for the one-dimensional case. For small $\tau_{\text {auto }} / \tau_{b}$, the pairwise vortex coalescence seems to play a role in the case where only one EPW is present. Large $\tau_{\text {auto }} / \tau_{b}$ diffusion corresponds to a different situation: the electrostatic potential slowly changes and the particle dynamics evolves in an adiabatic way.

Particles interacting resonantly with large electric field can be trapped and subsequently detrapped when instantaneous separatrices fluctuate. The long-time dynamics of such particles become chaotic throughout a large region of phase space when repeated wave-particle interactions occur. Such processes are relevant of adiabatic invariance theory and separatrix-crossing theory as presented in Refs. [18-20] for Hamiltonian systems and are beyond the realm of quasilinear theory.

We keep spatial periodicity on the system and we now discuss the long-time dynamics associated with multiple successive wave-particle interactions for SRS-F. An early study (1992) of this system (see Ref. [14] for more details) analyzed the time behavior of various wave actions, together with contribution of the trapped electrons to plasma wave action, for a little more than the time for a single interaction cycle of wave interaction, i.e., a little past the first maximum of pump action (partial) recovery. The electron plasma that was used was $95 \%$ composed of a Maxwellian distribution with a $15 \mathrm{keV}$ temperature, together with a 5\% Maxwellian hot contribution at a temperature of $100 \mathrm{keV}$. In this section we carry this simulation over many cycles and show the subtle effect of the hot-electron population. To make these hot-electron effects stand out we will first discuss the results without a hot-electron population.

\section{A. Plasma behavior without a hot-electron population}

Numerical simulations show that coherent particle trapping vortices are first created during the first few waveparticle interaction cycles due to strong scattering. On a longer time scale, the dramatically altered distribution function relaxes slowly via particle diffusion, with particles now distributed uniformly throughout phase-space region located near separatrices (and in particular inside the trapping region in phase space).

We begin with a simulation of SRS-F at high plasma temperature $T_{e}=15 \mathrm{keV}$, high enough for electron Landau damping to subdue rapidly growing but here unwanted backward SRS instability, in order to analyze in detail SRS-F alone. The convenient wave-vector choices are mode 2 for the EPW (i.e., $k_{F}=2 \Delta k=1.20 \omega_{p} / c$ ) and mode 4 for the pump, with the forward scattered mode number $k_{s F}$ being therefore $(4-2) \Delta k$, i.e., mode 2 .

The corresponding frequency is then $\omega_{s F}=\left(\omega_{p}^{2}+k_{s F}^{2} c^{2}\right)^{1 / 2}$ $\simeq 1.562 \omega_{p}$. For an electron temperature of $15 \mathrm{keV}$, the EPW frequency is close to $\omega_{F}=\left(\omega_{p}^{2}+3 k_{F}^{2} v_{\mathrm{th}}^{2}\right)^{1 / 2} \simeq 1.061 \omega_{p}$ and the mismatch in frequency is then $\delta \omega=\omega_{0}-\omega_{s F}-\omega_{F} \simeq$ $-0.023 \omega_{p}$.

The pump electric field is $e E_{0} / m_{e} \omega_{p} c$ to be 0.28 , which gives a quiver momentum of $a_{0}=0.108$, which corresponds to the product of an intensity times the pump wavelength squared of $1.6 \times 10^{16} \mathrm{~W} \mathrm{~cm}^{-2}$. We have here $k_{F} \lambda_{D} \simeq 0.205$.
In order to trigger the SRS-F instability in the Vlasov code (which is essentially noiseless), the electron distribution function was initially set to give a forward-propagating longitudinal wave at the onset (at least on the initial phase of the instability),

$$
\begin{aligned}
f\left(x, p_{x}, t=0\right) & =F_{\max }\left(p_{x}-m_{e} c \varepsilon \beta_{\mathrm{th}} \cos k_{F} x\right) \\
\times & {\left[1+\varepsilon \beta_{\mathrm{th}} \frac{k_{F} c}{\omega_{p}} \cos k_{F} x\right] . }
\end{aligned}
$$

Here $F_{\max }\left(p_{x}\right)$ is the Maxwellian distribution function and $\beta_{\mathrm{th}}=v_{\mathrm{th}} / c$ is the normalized thermal velocity. Note that, in the work of Skoric et al. in Ref. [2], where the threeoscillator model was used, the system is driven toward a spatial temporal chaos by just increasing the pump strength. Here $a_{0}$ is kept fixed and the plasma state should be contrasted with the picture of weak turbulence since SRS-B is here forbidden and the SRS-F instability is connected to a higher value of the phase velocity of the EPW. When the EPW amplitude is high enough (here $e E_{x, \max } / m_{e} c \omega_{p} \sim 0.12$ ) there exists a large class of resonant particle trajectories which can be trapped in the wave and may lead to a kinetic regime of the instability even if the parameter $k_{F} \lambda_{D}$ is taken under the value of 0.3 (usually established when the longitudinal field is weak). In slowly varying Hamiltonian system, it is the successive separatrix crossing by particles which may now lead to a chaotic state.

Bruhwiler and Cary in Ref. [20] showed that the adiabatic invariance is indeed broken because the trajectories of resonant particles cross a separatrix in phase space (specially at the $X$ point). They have used separatrix-crossing theory to evaluate the adiabatic invariant after a particle has crossed the separatrix, given the adiabatic invariant before the crossing. Before and after the crossing, adiabatic invariance theory may be used and close to the separatrix, it is then necessary to estimate the change in adiabatic invariant. Because the breakdown of adiabatic invariance occurs usually over the small regions of phase space where separatrix crossing is made, one could expect that explicit accounting for separatrix crossing to wave action would allow better formulation of wave action when such nonadiabatic kinetic effects are included. (This certainly demonstrated for at least the first action coupling cycle in Ref. [14].)

We choose to begin with a simulation in which SRS-F is generated in a standard fluidlike regime. The time behavior of the action densities for SRS-F is shown in Fig. 6, together with the relevant density action sum: the electromagnetic pair $S_{0}, S_{S F}$ and $C_{S F}=S_{0}+S_{S F}$ on the top panel and the pump and (lossy) electrostatic longitudinal actions $S_{0}, S_{F}$, and $C_{F}$ $=S_{0}+S_{F}$ on the bottom panel. The general behavior is as expected from the three-oscillator model: nonlinear and decaying oscillatory transfer from the pump to daughters, i.e., EPW $\left(\omega_{F}, k_{F}\right)$ plus Stokes $\left(\omega_{s F}, k_{s F}\right)$ modes and back again. Kinetic effects are weak with our choice of physical parameters and the system exhibits only three waves: however fast oscillations, but remaining at weak level, can be clearly seen in the electrostatic action density.

Figure 7 shows what happens in phase space. The top panel shows the growth of the EPW in the SRS-F process, 


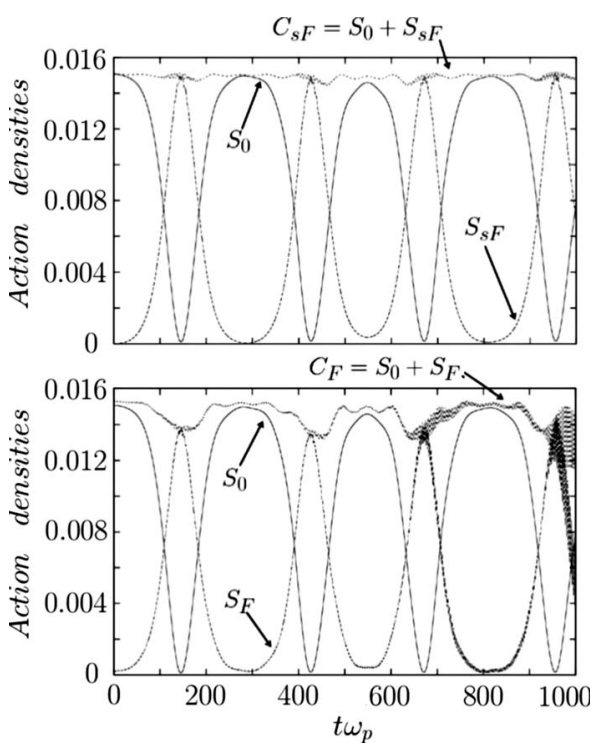

FIG. 6. For fluid phase of forward SRS, the time evolution of the different electromagnetic action densities is shown on the top panel while the second Manley-Rowe invariant related to the sum of the pump $S_{0}$ plus the EPW contribution is shown on the bottom panel. Here the physical parameters for SRS-F are $a_{0} \simeq 0.108$ and $T_{e}=15 \mathrm{keV}$ without any hot particle population.

showing impressive correlations with simple orbit theory. As expected, a fluidlike regime is observed since no particle trapping occurs here with our choice of physical parameters. Note that a small quantity of particles enters in the trapping region by crossing the lower separatrix at the $X$ point at time $t \omega_{p}=140$. After four particle-wave interaction periods (i.e., starting at $t \omega_{p} \simeq 900$ ), the electrostatic field increase and particle again are slowly accelerated. However we may now observe a more complex (stochastic) behavior when particles approach the separatrix $X$ point.

\section{B. Plasma behavior with hot-electron contribution}

We choose now to enhance the wave-particle interaction by introducing initially a small population of hot electrons $(5 \%)$ at the hot temperature of $T_{h}=100 \mathrm{keV}$. The hot temperature was high enough to ensure sufficient electrons to study particle trapping effect around the phase momentum $p_{\varphi F}$ without any initial beam-plasma instability from electrons accelerated by SRS-B (the major part of the distribution function is constituted by $95 \%$ of "cold" electrons at temperature of $T_{e}=15 \mathrm{keV}$ ). The role of this small hotelectron population is then to take into account the kinetic effects induced by SRS-B without introducing any another EPW. Here the simulations were performed on a long-time evolution in order to study the occurring of chaotic behavior of plasma.

The electromagnetic action densities $S_{0}, S_{s F}$, and $S_{\text {kin }}$ (for the kinetic part) are in the top panel and $S_{0}$ and $S_{F}$ are in the bottom panel in Fig. 8, together with their mutual sum $C_{S F}$ $=S_{0}+S_{S F}$ and $C_{F}=S_{0}+S_{F}+S_{\text {kin }}$. Thus considering the case of a single electrostatic wave $\left(\omega_{F}, k_{F}\right)$, stochasticity may be now connected with the complex dynamics of the particles mov-

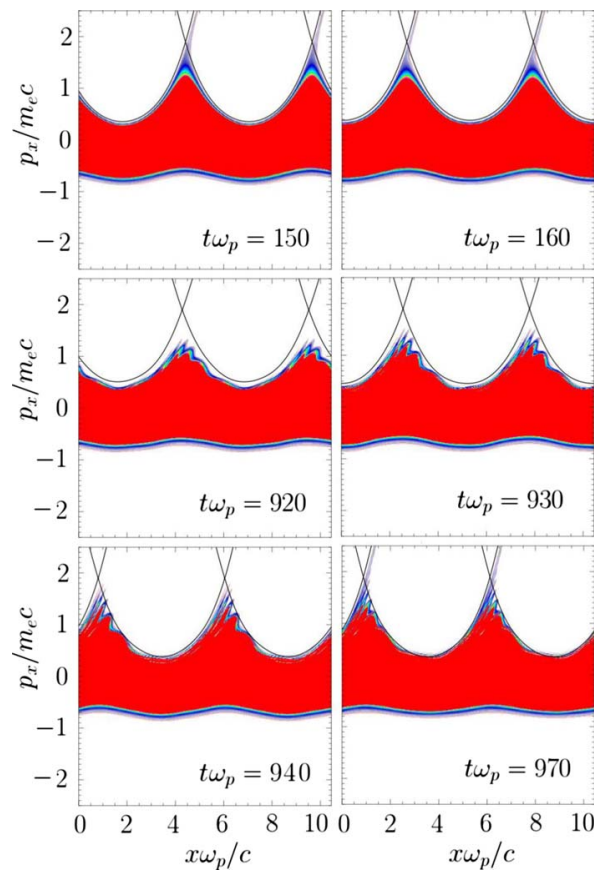

FIG. 7. (Color online) Phase space snapshots corresponding to the Manley-Rowe diagnostics shown in Fig. 6. The fluid phase of SRS-F is clearly observed since no particle is trapped. We observe impressive correlations at time $t \omega_{p}=130$, and after that time, with simple orbit theory and in particular concerning the lower separatrix limit. After several particle-wave interaction cycles (middle and bottom panels), a more complex state is observed as the result of slowly fluctuating separatrices and in particular located toward the $X$ point.

ing across the separatrices in the so-called stochastic layer. When separatrices fluctuate slowly (in an adiabatic manner), particles located close the separatrices can escape from the wave potential trough where they are trapped, inversely, they can become detrapped, or they can be caught again by the trough (retrapping). The general behavior for the electromagnetic actions is as expected with nonlinear oscillatory action transfer from mother wave (pump) to daughter waves and back again. Note that the electromagnetic sum $C_{s F}$ is well conserved during the simulation. It is not the case of the pump plus EPW action which decreases (not shown here). This is clearly the signature of kinetic effects due to waveparticle interaction involving the EPW that can accelerate particles and thus lose action. This plasma wave action loss behaves nonlinearly and is related to a significant particle trapping. Taking into account the relativistic kinetic energy of all electrons above the lower separatrix (and dividing by $\omega_{F}$ to obtain an action density) in the sum $C_{F}$ would account of the missing action. Here

$$
S_{\text {kin }}=\frac{W_{\text {kin }}}{\omega_{F}}=\frac{m_{e} c^{2}}{\omega_{F} L} \int_{0}^{L} d x \int_{p_{\text {low }}}^{+\infty}(\gamma-1) f\left(x, p_{x}, t\right) d p_{x} .
$$

It is clear in Fig. 8 (bottom panel) that adding $S_{\text {kin }}$ to $S_{0}$ $+S_{F}$ gives now a good action conservation during the first cycles of the wave-particle interaction. It should be noted that such a hypothesis to determine $S_{\text {kin }}$ is valid only when 

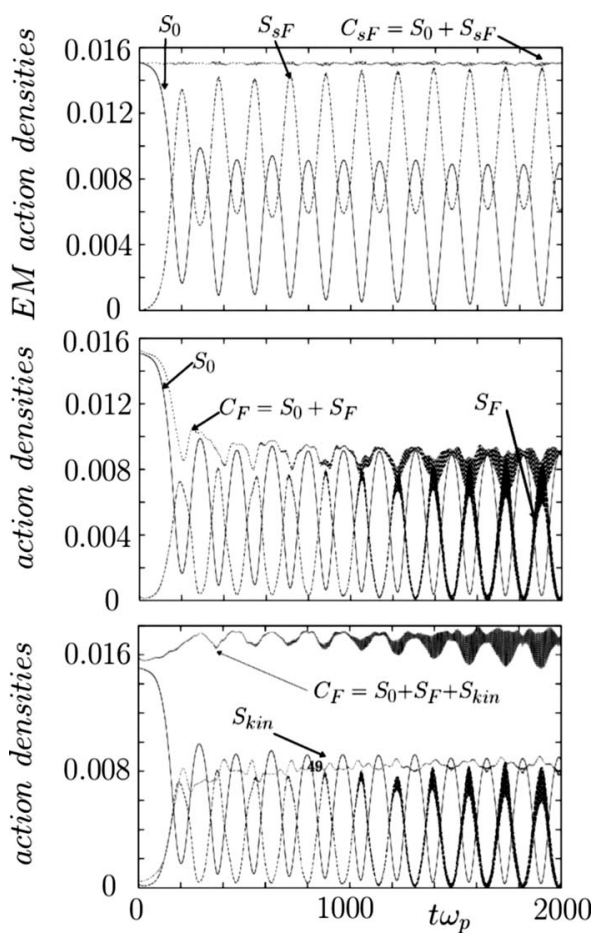

FIG. 8. For kinetic regime of forward Raman scattering, the time evolution of the electromagnetic action densities in the top panel over a long time: $S_{0}$ for the pump, $S_{S F}$ for the Stokes mode, and the first Manley-Rowe invariant $C_{S F}=S_{0}+S_{F}$ showing a very good conservation over a long time. The bottom panel exhibits the corresponding action transfer to fast (or trapped) particles. The Kubo number is here close to $\tau_{\text {auto }} / \tau_{b} \simeq 55$ indicating that particle diffusion is due to the successive particle trapping-detrapping and retrapping processes.

the accelerating waves are well defined and distinct (as here). We note also that the possibility of an anti-Stokes scattering resulting from nonlinear coupling (with matching conditions such that $\omega_{0}=\omega_{a s F}-\omega_{F}$ and $\left.k_{0}=k_{a s F}-k_{F}\right)$ is also possible. From the linear dispersion relation of electromagnetic wave, the frequency and wave vector of the anti-Stokes mode are then given by $\omega_{a s F} \simeq 3.736 \omega_{p}$ for $k_{a s F} c / \omega_{p}=3.6$ (or equivalently mode 6). Using the corresponding frequency and wave vector of the EPW generated by the direct forward Raman scattering, i.e., $\omega_{F} \simeq 1.061 \omega_{p}$ and $k_{F} c / \omega_{p}=1.2$ (i.e., mode 2 ), the mismatch in frequency is close to $\delta \omega=\omega_{a s}-\omega_{0}-\omega_{F}$ $\simeq 0.075 \omega_{p}$.

If one looks at the top panel and the bottom panel of Fig. 8 , it is clear that the essential reason is that about (0.0150.01 ), i.e., 0.007 action units have been irreversibly transferred to the kinetic action component of the plasma wave. After that, the system is almost (but not quite) reversible, with a slight additional irreversible transfer up to the fourth cycle at about $t \omega_{p} \simeq 800$. The oscillations on the value after that probably reflect the slight variations in particles classed or nontrapped as the plasma wave amplitude changes which are not really trapped or detrapped but only appear to be so because the wave amplitude is varying somewhat too quickly for the asymptotically slow trapping approximation to be truly valid. The cause of the very rapid variations visible at very late times, whose amplitude seems to be increasing, is not yet clear.

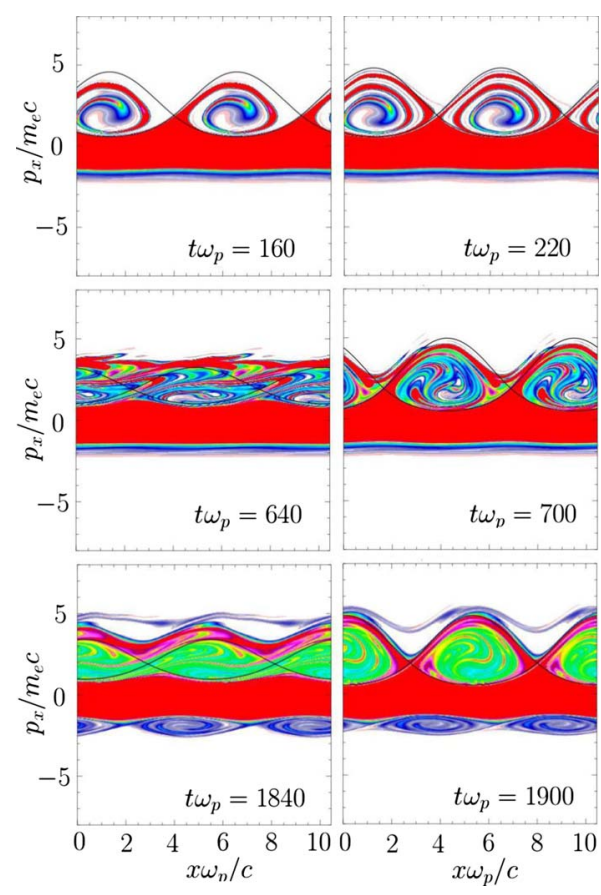

FIG. 9. (Color online) For SRS-F scenario in the kinetic regime at high $\tau_{\text {auto }} / \tau_{b}$ values, phase-space snapshots of the electron distribution function in the first wave-particle interaction cycle on the top panels. Particle trapping is now observed with spiraling thin filaments inside the trapping structures. On bottom panels, long-time evolution after multiple wave-particle interaction cycles. An electron beam-acoustic mode is observed around $p_{\varphi F}$, while (anomalous) particle diffusion takes place inside the trapping structures.

In that simulation, we have $\tau_{\text {auto }} / \tau_{b}$ which is given by the product of $\left(\omega_{p} / \Delta \omega\right)$ by $\sqrt{\left(e E_{x, \max } / m_{e} \omega_{p} c\right)\left(k_{F} c / \omega_{p}\right)}$ close to $1 / 0.020 \sqrt{0.10 \times 1.20} \simeq 54.7 \gg 1$. Thus for $\tau_{\text {auto }} / \tau_{b} \gg 1$, the potential dynamics slowly evolves in an adiabatically way leading to weak diffusion due to the fact that the separatrices may fluctuate.

Next we examine the changes with time of the electron distribution function in phase space, as shown in Fig. 9. We have also plotted the separatrix limits, calculated through the data of the electric potential given by the code.

During the first temporal cycle, inside the interval $\left[160,220 \omega_{p}^{-1}\right]$, the system is not stochastic and is driven by a filamentary structure as particles spiraling smoothly around the local $O$ point, which develops inside the wave potential. In the top panels in Fig. 9 at the times indicated the plasma wave amplitude is near its maximum and the deeply trapped electrons have done about two and a half oscillations, judging by the two and half rotation of the single trapping spiral "tendril" in each separatrix cell. We see clearly that the filaments get thinner as time goes on. The filamentation described here results from the parametric instability and mixes "particles" in the neighborhood of the lower separatrix limit (note that thin filament disappears at time $t \omega_{p}=220$ when located just at the separatrix) when the longitudinal electric field decreases. Thus these particles, initially trapped in the potential well, experience a slowly pulsating lower separatrix and become detrapped and fall in the bulk population. Such a behavior, which is not connected to a subgrid-scale filamen- 
tation of the Vlasov equation but to the chaotic trappingdetrapping process, may lead to an irreversible process. Note that this trapping-detrapping scenario arises over large phase-space scale, well above the "numerical scale" imposed by the phase-space cell $\Delta x \Delta p_{x}$. The middle panels in Fig. 9 show the electron dynamics after three cycles when the electric field begins to increase in the interval $\left[640,700 \omega_{p}^{-1}\right]$. Instead of spiraling around the $O$ point as before when the wave was growing, the phase space exhibits a more complex structure. After about three coupling cycles, judging by the first left-hand side frame with fairly low field, one sees that the nearly free-flow convection, while the electrostatic field was low, has resulted in the overlap of two of the original trapping cells. In particular the transporting particle of the first trapping structure may now reach the second vortex leading to large scale chaos by overlap. One is clearly seeing the result near-ballistic free flow alternated with stages of these being rolled up vortically in trapping cells. By the latest times this has resulted in cells with rather uniformly distributed mostly trapped distributions inside upper layers of nearly untrapped passing particles and the untrapped thermal distribution below. Only a few such cycles suffice to produce a distribution function that experiences phase mixing (and leading to particle diffusionlike process by phase-space mixing) under further cycles of trapping, detrapping, and retrapping.

If the initial momentum of particles is high enough (i.e., greater than the phase momentum $p_{\varphi F} \simeq 1.92 m_{e} c$ ) particles will uncover a large field and become accelerated, crossing the separatrix from above. Depending on its phases, the particles will either be detrapped and crossed the upper separatrix leading to an accelerated beam [and will contribute to the beam-acoustic mode (BAM) as observed in particle-incell (PIC) simulation in Ref. [21]] or below the separatrix as in the previous case.

Bottom panels in Fig. 9 show what happens after ten cycles. On a longer time scale, the dramatically altered distribution function seems to relax slowly (but a plateau is not observed here), with particles now distributed uniformly inside the vortex in the central region around the $O$ point in a coarse-grained sense. After several cycles, in the asymptotic limit the distribution function changes little under further cycles. The other point to note is the presence of lower layer of weaker trapping cells, indicating a plasma wave going to the left with about the same phase velocity in the opposite direction but with a rather lower field. Perhaps it is this beating of this with the main wave that is the cause of the rapid modulation seen to begin at about $t \omega_{p}=1100$. However on a long time an anti-Stokes scattering may be excited by wave coupling and the corresponding EPW may be observed at (negative) phase momentum $p_{\varphi} \simeq-1.9 m_{e} c$. As predicted by recent studies, the EPW generated in the SRS-F process evolves from the initial Langmuir waves to electron BAMs as electron trapping modifies the distribution to a nonMaxwellian form. Another feature is the particle diffusion. The microscopic key to this evolution toward a smoother distribution function around $p_{\varphi F}$ is the chaotic dynamics of particles moving in the vicinity of the separatrices (by trapping-detrapping processes). We see clearly in Fig. 9, in bottom panels, that phase-space holes are filled up. Succes- sive trapping-detrapping and retrapping processes in the case of a single pulsating plasma wave are chaotic and lead to an anomalous particle diffusion. While the diffusion may be quasilinear for small $\tau_{\text {auto }} / \tau_{b}$, large $\tau_{\text {auto }} / \tau_{b}$ diffusion is here due the successive crossing of the separatrices and does not involve Gaussian distribution.

\section{PARTICLE-TEST DIAGNOSTICS}

In order to point out and explain the role of the stochastic processes at work in the wave-particle interactions and to shed light on their influence on the particle diffusion, we have used particle test diagnostics. The trajectories of test particles have been obtained by integrating the equations of characteristics,

$$
\begin{gathered}
\frac{d x}{d t}=v_{x}=\frac{p_{x}}{m_{e} \gamma}, \\
\frac{d p_{x}}{d t}=F\left(x, p_{x}, t\right)=e\left(E_{x}+\frac{p_{x} B_{z}}{m_{e} \gamma}\right) .
\end{gathered}
$$

The force $F$ is directly computed from the results of the Vlasov simulation. These investigations also provide a powerful technique to analyze the diffusion mechanism because the fields and the trajectories of the test particles are free from the statistical fluctuations which are characteristic of the PIC codes. The test particles are initially located in five narrow bands equally spaced around the phase momentum $p_{\varphi F} \simeq 2 m_{e} c$ (their total spread again is about $\Delta p_{\varphi F} \simeq 2 m_{e} c$ ). By following the trajectories of 5000 particles, we compute several statistical quantities. Here we focus on the following quantities, $\sigma_{p}^{2}=\left\langle\Delta p^{2}\right\rangle-\langle\Delta p\rangle^{2}$ (top panel), and the Kurtosis factor for the momentum variable, $K_{p}=\left(\left\langle\Delta p^{4}\right\rangle\right.$ $\left.-\langle\Delta p\rangle^{4}\right) / 3\left(\left\langle\Delta p^{2}\right\rangle-\langle\Delta p\rangle^{2}\right)^{2}$. The angular brackets $\left\langle\Delta p^{n}\right\rangle$ $=\frac{1}{N} \sum_{i=1}^{N}\left[p_{i}^{n}(t)-p_{i}^{n}(0)\right]$ denote an average over all test particles and $K_{p}=1$ is the value for a distribution which is Gaussian in momentum, higher values of $K_{p}$ corresponding to a distribution function relatively more peaked than a Gaussian.

We recall that the time derivative of the quantity $\sigma_{p}^{2}$ in quasilinear theory is proportional to the quasilinear diffusion coefficient, $\mathcal{D}_{\mathrm{ql}}=0.5 d \sigma_{p}^{2} / d t$. However, we are here in the case of a large values of $\tau_{\text {auto }} / \tau_{b}\left(\tau_{\text {auto }} / \tau_{b} \simeq 57.4\right)$ with a large but not infinite correlation time between particles (the potential encountered by particles is not static), characterized by a quasiadiabatic dynamical evolution; therefore a diffusive quasilinear behavior is not a priori expected to hold (see Ref. [22]). Moreover, we are here involved with only one electrostatic mode, $\left(\omega_{F}, k_{F}\right)$, which of course prevents from the possibility of averaging over many random phases (we are in the singular limit of a peaked spectrum, i.e., a Dirac delta function, thus with only one phase). This restriction to a limited set of initial conditions for particles (which in our case are all in the resonance band around the single-mode velocity phase, $p_{\varphi F} \simeq 2 m_{e} c$ ) is known to lead more likely to a Levy flight picture rather than a diffusive behavior [22].

We sketch here some preliminary results agreeing with this interpretation. In Fig. 10 we plot the time evolution of $\sigma_{p}^{2}$ and $K_{p}$ for a Vlasov simulation started with a warmer elec- 


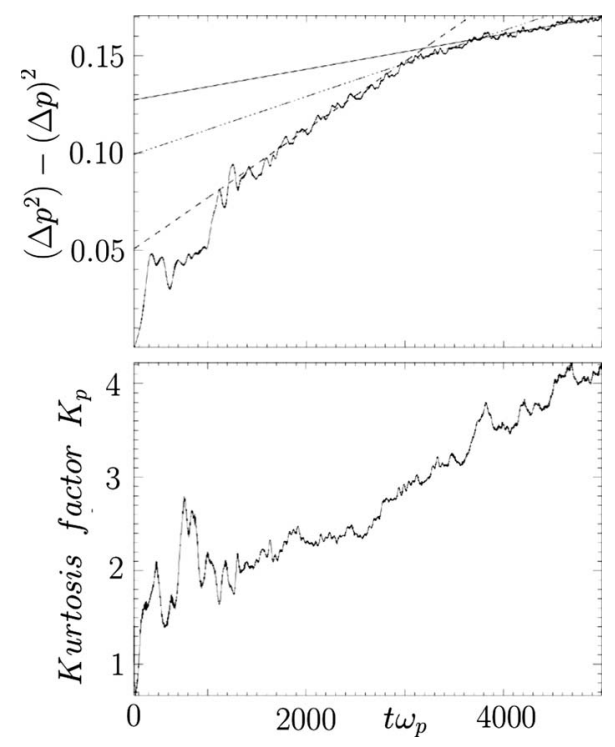

FIG. 10. To shed light on their influence on the particle diffusion, we have used particle test diagnostics. The trajectories of test particles have been obtained by integrating the equations of characteristics using the fields given by the Vlasov code. We compute several statistical quantities. Here we focus on the following quantities: $\sigma_{p}^{2}$ on the top panel and the Kurtosis factor on the bottom panel.

tron population corresponding to $15 \%$ of the total one (with a hot population of $100 \mathrm{keV}$ ).

In Fig. 11 we show the time behavior of some relevant action densities (see paragraph 6) for comparison with the time scales of the forward Raman-scattering process. The squared variance of the particles' momentum spread, $\sigma_{p}^{2}$,

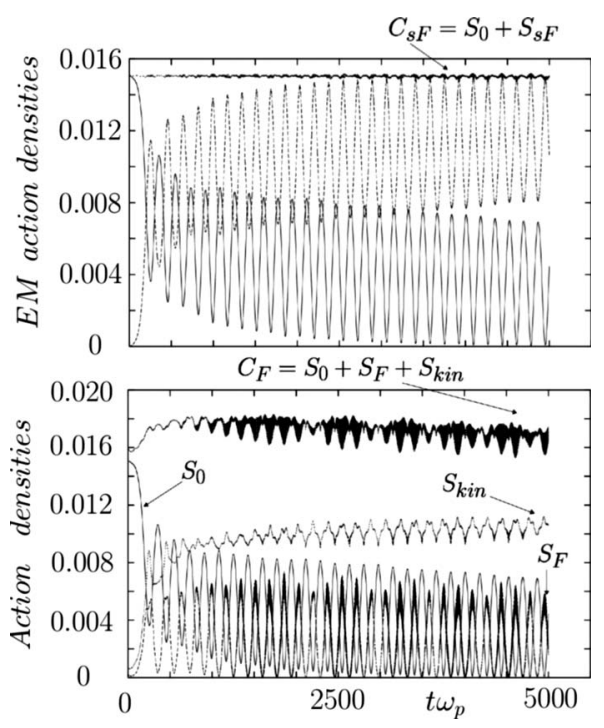

FIG. 11. For the same simulation parameters as in Fig. 10, the time evolution of the electromagnetic action densities (in the top panel) over a long time: $S_{0}$ for the pump, $S_{s F}$ for the Stokes mode, and the first Manley-Rowe invariant $C_{S F}=S_{0}+S_{F}$ showing a very good conservation over a long time. The bottom panel exhibits the corresponding action transfer to fast (or trapped) particles on a long time for several wave-particle cycles. clearly displays a nonlinear temporal dependence. A quite complex behavior, characterized by important fluctuations, is recognized in the initial phase (approximately up to $t \omega_{p}$ $\simeq 1000$, corresponding about to only four oscillations of the forward Raman action mode), which is dominated by vortex development and vortex interactions inside the phase-space region of trapped particles. This kind of dynamics interplays with the separatrix oscillations and the phenomena of particle crossing (see paragraph $\mathrm{V}$ ), which eventually lead to a decrease in the diffusion process. Over longer times, saturation is achieved (at $t \omega_{p} \simeq 2500$ the action densities have undergone about 13 oscillations) and the usual quasilinear behavior is recovered. Notice that only the superimposed dashed line of Fig. 10 should be interpreted as a signature of a quasilinear behavior in the interval $\left[4000,5000 \omega_{p}^{-1}\right]$ since it occurs over a time at which saturation is expected (the electromagnetic and electrostatic modes have been interested in a "sufficient" number of energy exchanges) and since it agrees with a dampening of the diffusion process. The other two lines, through their slope, should be taken just as localin-time estimations of the efficient diffusion coefficient, let us say $\mathcal{D} *$, within a restricted time interval [where the line fits the "mean tangent" to $\left.\sigma_{p}^{2}(t)\right]$. For the case of the dashed and point-dashed lines of Fig. 10 we have estimated the two respective values $\mathcal{D}_{1}^{*} \simeq 0.0000175\left(m_{e} c\right)^{2} \omega_{p}$ and $\mathcal{D}_{2}^{*} \simeq 0.0000085\left(m_{e} c\right)^{2} \omega_{p}$. For the dashed line we argue it to asymptotically approach the quasilinear value, which therefore we roughly estimate as $\mathcal{D}_{\mathrm{ql}} \leqq \mathcal{D}_{3}^{*}$ $\simeq 0.0000045\left(m_{e} c\right)^{2} \omega_{p}$. Finally, the graphics of $K_{p}(t)$ (bottom panel in Fig. 10) indicates that the test-particle distribution function undergoes a quite complicate rearrangement during the stage of the Raman processes dominated by vortex dynamics in the phase space $\left(t \omega_{p} \lesssim 2500\right)$. Here we find a competition between narrowing and spreading processes in the momentum space [also notice that $K_{p}(t=0)>1$ since the test-particle distribution function in the momentum space initially consists of a collection of equally spaced delta functions]. At later time fluctuations become less important and $K_{p}$ increases almost linearly in time, according with the picture of an almost uniform acceleration mechanism which brings particles to reach almost the same speed (thus damping the diffusion process and peaking again the distribution function in the momentum space). Results not shown here also indicate that a saturation in $K_{p}$ can be achieved at later times, which therefore corresponds to a true quasilinear regime, where the test-particle distribution function does not change anymore in average $\left(K_{p} \simeq\right.$ const) even if it has achieved a non-Gaussian shape $\left(K_{p} \neq 1\right)$ due to the particle acceleration.

Figure 11 shows the corresponding action conservation of the Manley-Rowe partition. This Vlasov simulation was carried out for many (29) cycles of wave-particle interaction. For several interaction cycles Fig. 11 exhibits the same oscillatory behavior we have met in Fig. 8 in Sec. V B. However it must be pointed out that a slight increase in the kinetic action transfer takes place after the first interaction cycles. In addition to the action transfer to fast particle during the first interaction cycle, successive cycles lead to a slight second enhanced action transfer process on a long time. This irreversible growth is not related to the entropy 
production of the (numerical) subgrid-scale filamentation but to the chaotic trapping-detrapping process.

Several other tests at the varying of the relevant parameters have been run, but the critical dependence on the initial spread of the test-particle distribution function deserves a more detailed discussion which is beyond the purposes of this paper and which will be presented in a following study.

\section{CONCLUSION AND OUTLOOK}

We have investigated the stimulated Raman scattering in conditions relevant to the kinetic regime of the instability characterized by high value of the product of the wave number of the EPW by the Debye length $k \lambda_{D}$. Our study includes relativistic Vlasov-Maxwell simulations and Manley-Rowe diagnostics, phase-space representation with simple orbit theory and test-particle calculations. The emphasis of the study is in successive relativistic wave-particle interactions on the long time, which show many interesting features. While particles experience a nonlinear trapping mechanism scenario during the first cycles of wave-particle interaction, the long-time evolution of $f$ differs according to the type of instability. For a small value of the parameter $\tau_{\text {auto }} / \tau_{b}$ (here SRS-B scenario), the simulations show that the main saturation process of SRS-B is provided by a process which begins with pairwise trapping structure merging of phase-space holes, followed by the occurring of secondary rescattering instabilities in the regime of $k_{B} \lambda_{D} \gtrsim 0.27$.

In the SRS-F scenario, the phase velocity of the EPW is higher and falls in a region of very low density; the stochastic transitions performed by resonant particles determine now the long-time evolution of the plasma. This case corresponds to a higher value of the Kubo-type number $\tau_{\text {auto }} / \tau_{b}$. Indeed the trapped particles in the EPW potential well near the separatrix may be detrapped (and become passing particles) or even retrapped in the vicinity of the separatrices. Such transitions of the particles are connected to the slowly fluctuating character of the longitudinal field which is oscillating during the self-consistent wave-particle interaction in an adiabatic manner. This may lead to a Hamiltonian stochasticity near the separatrix limits.

\section{ACKNOWLEDGMENTS}

The authors acknowledge the IDRIS (Institut du Développement et des Ressources pour l'Informatique Scientifique, CNRS, Orsay, France) for computer time allocation on their supercomputers. The authors are grateful to P. Bertrand, V. Latocha, and S. Labrunie for valuable collaborations and helpful discussions. The authors are grateful to T. W. Johnston for fruitful discussions and a critical reading of the paper.
[1] T. Umeda and T. Ito, Phys. Plasmas 15, 084503 (2008).

[2] M. M. Skoric, M. S. Jovanovic, and M. R. Rajkovic, Phys. Rev. E 53, 4056 (1996).

[3] J. L. Kline, D. S. Montgomery, B. Bezzerides, J. A. Cobble, D. F. DuBois, R. P. Johnson, H. A. Rose, L. Yin, and H. X. Vu, Phys. Rev. Lett. 94, 175003 (2005).

[4] M. Albrecht-Marc, A. Ghizzo, T. W. Johnston, T. Reveille, D. Del Sarto, and P. Bertrand, Phys. Plasmas 14, 072704 (2007).

[5] G. T. Morales and T. M. O’Neil, Phys. Rev. Lett. 28, 417 (1972).

[6] F. Huot, A. Ghizzo, P. Bertrand, E. Sonnendrucker, and O. Coulaud, J. Comput. Phys. 185, 512 (2003).

[7] D. S. Montgomery, R. J. Focia, H. A. Rose, D. A. Russell, J. A. Cobble, J. C. Fernandez, and R. P. Johnson, Phys. Rev. Lett. 87, 155001 (2001); Phys. Plasmas 9 (5), 2311 (2002).

[8] R. E. Turner, K. Estabrook, R. P. Drake, E. A. Williams, H. N. Kornblum, W. L. Kruer, and E. M. Campbell, Phys. Rev. Lett. 57, 1725 (1986).

[9] P. Bertrand, A. Ghizzo, S. J. Karttunen, T. J. H. Pattikangas, R. R. E. Salomaa, and M. Shoucri, Phys. Plasmas 2 (8), 3115 (1995).

[10] H. X. Vu, L. Yin, D. F. Dubois, B. Bezzerides, and E. S. Dodd, Phys. Rev. Lett. 95, 245003 (2005).

[11] L. Yin, W. Daughton, B. J. Albright, B. Bezzerides, D. F. DuBois, J. M. Kindel, and H. X. Vu, Phys. Rev. E 73,
025401(R) (2006).

[12] I. B. Bernstein, J. M. Greene, and M. D. Kruskal, Phys. Rev. 108, 546 (1957).

[13] A. Ghizzo, P. Bertrand, J. Lebas, T. W. Johnston, and M. Shoucri, Phys. Plasmas 3, 650 (1996).

[14] T. W. Johnston, P. Bertrand, A. Ghizzo, M. Shoucri, E. Fijalkow, and M. R. Feix, Phys. Fluids B 4, 2523 (1992).

[15] P. Bertrand, A. Ghizzo, M. R. Feix, E. Fijalkow, P. Mineau, N. D. Suh, and M. M. Shoucri, in Proceeding of the International Workshop on Nonlinear Phenomena in Vlasov Plasmas, Cargèse, France, 11-16 July 1988, edited by F. Doveil (Editions de Physique, Orsay, 1989), p. 109.

[16] A. Ghizzo, B. Izrar, P. Bertrand, E. Fijalkow, M. R. Feix, and M. M. Shoucri, Phys. Fluids 31, 72 (1988).

[17] P. Bertrand, A. Ghizzo, S. J. Karttunen, T. H. Pattikangas, R. R. E. Salomaa, and M. M. Shoucri, Phys. Fluids B 4, 3590 (1992).

[18] J. R. Cary, D. F. Escande, and J. L. Tennyson, Phys. Rev. A 34, 4256 (1986).

[19] J. R. Cary and R. T. Skodje, Phys. Rev. Lett. 61, 1795 (1988).

[20] D. L. Bruhwiler and J. R. Cary, Phys. Rev. E 50, 3949 (1994).

[21] L. Yin, B. J. Albright, K. J. Bowers, W. Daughton, and H. A. Rose, Phys. Plasmas 15, 013109 (2008).

[22] D. F. Escande and F. Sattin, Phys. Rev. Lett. 99, 185005 (2007). 